\title{
Association of Skeletal and Dental Maturity Indicators with the Onset of Menarche and Its Applicability for Growth Modification of Jaw Bases in Females Aged between 7 and 14 Years
}

\author{
Swapnil Korde ${ }^{1}$, Pallavi Daigavane 2 , Prutha Ganesh Khakhar 3 , Priyanka Niranjane ${ }^{4}$, Bhagyashree Chimote \\ ${ }^{1}$ Department of Orthodontics, Sharad Pawar Dental College, Sawangi, Wardha, Maharashtra, India. \\ ${ }^{2}$ Department of Orthodontics, Sharad Pawar Dental College, Sawangi, Wardha, Maharashtra, India. \\ ${ }^{3}$ Department of Orthodontics, Sharad Pawar Dental College, Sawangi, Wardha, Maharashtra, India. \\ ${ }^{4}$ Department of Orthodontics, Sharad Pawar Dental College, Sawangi, Wardha, Maharashtra, India. \\ ${ }^{5}$ Department of Prosthodontics, VYWS College, Amravati, Wardha, Maharashtra, India.
}

\section{ABSTRACT}

\section{BACKGROUND}

Orthodontic diagnosis and treatment planning for skeletal problems in growing children must involve growth prediction. Apart from the knowledge of chronological, dental and skeletal age of a growing patient, it is also mandatory to predict growth and its outcome during the treatment. In females, onset of menarche is a sign of pubertal growth and a biological skeletal maturity indicator. Studies have shown that there is an association amongst skeletal and dental maturity indicators. This study was therefore designed to estimate association between skeletal- and dental-maturity indicators and onset of menarche in females and evaluate its applicability for growth modification in females.

\section{METHODS}

140 female patients were divided into 7 groups and each patient was asked about their menarcheal status. Radiographs such as orthopantomography, lateral cephalogram and middle phalanx of third finger were taken from each patient to assess the CVMI status, stages of dental calcification and MP3 stages.

\section{RESULTS}

The correlation between onset of menarche, CVMI status, stages of dental calcification and MP3 stages is of high statistical significance (p-0.0001).

\section{CONCLUSIONS}

There are strong correlations between all the parameters studied which signifies that assessment of skeletal maturation with any of the above methods studied, gives exact growth maturation age of the patient.

\section{KEY WORDS}

CVMI Status, MP3 Stages, Stages of Dental Calcification, Growth Prediction, Menarche

\section{Corresponding Author:} Dr. Prutha Ganesh Khakhar, Department of Orthodontics, Sharad Pawar Dental College Wardha, Maharashtra, India. E-mail:pruthakhakhar@gmail.com

DOI: $10.14260 / j e m d s / 2020 / 324$

Financial or Other Competing Interests: None.

How to Cite This Article:

Korde S, Daigavane P, Khakhar PG, et al. Association of skeletal and dental maturity indicators with the onset of menarche and its applicability for growth modification of jaw bases in females aged between 7 and 14 years. J. Evolution Med. Dent. Sci. 2020;9(18):1484-1489, $10.14260 / \mathrm{jemds} / 2020 / 324$

Submission 29-01-2020, Peer Review 17-04-2020, Acceptance 23-04-2020, Published 04-05-2020.
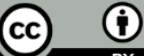


\section{BACKGROUND}

Balance and harmony of facial structures are the important factors in the making or masking of the beauty and character of the face. The field of orthodontics is indissolubly connected with the art of balancing the human face. 'Time' being the fourth dimension, is a key word in orthodontics, as it is one of the important factors for initiation of different treatment procedures in the growing patient. ${ }^{1}$ Authors have suggested that growth modulation treatment should be started at the beginning of the pubertal growth spurt. Prediction of optimal timing of treatment in Orthodontics and Dentofacial Orthopaedics is allied closely to the identification of stages of accelerated growth that can contribute meaningfully to the modification of skeletal disproportion in an individual patient.

Females often begin the process of puberty at a lower age than males. ${ }^{2}$ This signifies that females often will enter the pubertal growth spurt at a younger age suggesting that they demand treatment at an earlier point of time. Body height, ${ }^{3-5}$ skeletal maturation of hand and wrist, ${ }^{6-9}$ menarche and voice changes, ${ }^{10,11}$ are biological indicators of skeletal maturity. ${ }^{12}$ These Indicators primarily refer to somatic deviations at puberty, while the maxilla and mandible follow a pattern of growth that is intermediate between neural and somatic growth governed by genetic and epigenetic factors. The mandible follows the somatic growth curve more closely than the maxilla, which has a more neural growth pattern. ${ }^{13}$ Maturational status has large impact on diagnosis, treatment goals, treatment planning and the eventual outcome of orthodontic treatment. Due to individual growth variations, skeletal age assessment is vital in expressing viable customized orthodontic treatment plans.

Skeletal maturation staging with the help of radiographic analysis is a extensively used method to predict timing of pubertal growth, to estimate growth velocity and to estimate proportion of residual growth remaining. ${ }^{14}$ Skeletal maturation assessed on hand wrist radiographs is considered as the best indicator of maturity \& has been found to be meticulously related to the growth spurt. In an effort to decrease the radiographic exposure, assessment of maturity from the Hand-wrist radiographs has been additionally simplified by observation of progressive ossification of the MP3 (middle phalanx of 3rd finger) taken on a dental film ${ }^{15}$. This was modified later by Rajagopal \& Kansal (2002). This method is an accepted standard parameter, which predicts the timing of the pubertal growth spurt with the high degree of accuracy. 16

The utility of lateral cephalometric radiographs by reviewing the maturation of the cervical vertebrae to assess skeletal maturation has also been studied. This method, was first defined by Lamparski (1972) ${ }^{17}$ and explained by Hassel and Farman (1995).18 As compared to somatic growth, the calcification rate of the teeth is controlled more by genetics than by environment factors, with a lower variability. ${ }^{19,20}$ Studies done by Anderson et al $(1975)^{21}$ has shown that tooth development is independent of hormonal and nutritional factors. Other studies have shown that, age determination assessing dental maturity correlates to the chronological age in a greater extent than other methods of measuring the development, such as skeletal maturation, height and weight. ${ }^{22,23}$ Height and weight measurements are one of the powerful tools for growth assessment but are practically not useful in clinical orthodontics as it requires longitudinal data which is seldom available and needs time with repeated and periodic observations.

Dental age can be measured either by tooth eruption dates or by the development of tooth calcification. Studies done by Chertkow (1979) ${ }^{24}$ and (1980) 25 and Sierra AM (1987) ${ }^{26}$ showed that the phases of tooth mineralization of mandibular canine appear to link better with ossification stages than the other teeth.

Adolescence is the transitional period between the juvenile stage and adulthood, during which the secondary sexual characteristics appear, the adolescent growth spurt takes place and profound physiologic changes occur. Growth prediction in females using appearance of secondary sexual characteristics requires frequent physical examination and regular observational periods which are impractical in clinical orthodontic practice. Instead; history of onset of menstruation or menarche in females is a reliable indication suggesting that the maximum pubertal skeletal growth has been attained. Clinically, the effectiveness of dentofacial orthopaedic therapy can be reliant on all of these factors, but neither chronological age nor the level of dental development alone can reveal growth status of an individual, therefore satisfactory association with skeletal maturation indicators is essential to provide an acceptable basis for the timing of therapy.

The present study was planned to estimate the association of chronological age, skeletal maturity indicators (CVM status, MP3 stages) and dental maturity indicator (mandibular canine root calcification) with onset of menarche for assessment of pubertal growth spurts in female aged between 7 to 14 years and to evaluate its applicability for growth modification of jaw bases.

Informed consent was taken from the parents and Institutional consent was obtained for use of patient data The study was given approval by the Scientific and Ethical Committee of the institute.

\section{METHODS}

The present study was a cross-sectional study and conducted in the Department of Orthodontics, Sharad Pawar Dental College \& Hospital Sawangi (Meghe), Wardha between December 2017 to December 2019. The study population comprised of 140 screened and selected females of age group 7 to 14 years, visiting the outpatient department which were the bonafide patients of SPDC and Hospital. A detailed written informed consent was prepared and obtained from the parents of the subject to be included in the study

\section{Distribution of Groups}

Total sample size consisted of 140 females and were divided into seven groups i.e., from group $7-8$ as 8 to $13-14$ as 14 . The menarcheal status was asked to patients and was confirmed from the mother. Also, a close ended questionnaire was formulated and explained in the language of understanding and was given to parents which was regarding the menarche and physical development. 


\section{Radiographs Taken for Each Patient}

1. Lateral Cephalograms

For evaluating the morphologic features of the cervical vertebral bodies were outlined using the modified method of cervical vertebral maturation. ${ }^{12}$

2. Orthopantomogram (OPG)

Mandibular canine development stage was evaluated on an OPG according to stages for tooth development 23 .

3. Radiograph of middle phalanx of third finger (MP3) of left hand was used for assessing ossification stages of epiphysis and diaphysis. ${ }^{16}$

\section{Statistical Analysis}

After complete evaluation the readings were tabulated and subjected to statistical analysis which was done by using descriptive and inferential statistics using Chi square test and inter-rater reliability analysis. The software used in the analysis was SPSS 17.0 version and GraphPad Prism 6.0 version and $p<0.05$ was considered as level of significance.

\section{RESULTS}

\begin{tabular}{|cccc|}
\hline Menarche & No & Yes & $\times 2$-Value \\
Initiation $(\mathrm{n}=38)$ & 38 & 0 & \\
Acceleration $(\mathrm{n}=25)$ & 23 & 2 & \\
Transition $(\mathrm{n}=18)$ & 13 & 5 & $\mathrm{p}=0.0001, \mathrm{~S}$ \\
Deceleration $(\mathrm{n}=19)$ & 11 & 8 & \\
Maturation $(\mathrm{n}=15)$ & 0 & 15 & \\
Completion $(\mathrm{n}=25)$ & 4 & 21 & \\
\hline Table 1. Association of CVM Status with Menarche
\end{tabular}

\begin{tabular}{|cccc|}
\hline Menarche & Yes & No & *2-Value \\
F $(\mathrm{N}=20)$ & 0 & 20 & \\
FG $(\mathrm{N}=23)$ & 3 & 20 & 63.5 \\
$\mathrm{G}(\mathrm{N}=17)$ & 4 & 13 & $\mathrm{p}=0.0001, \mathrm{~S}$ \\
$\mathrm{H}(\mathrm{N}=20)$ & 8 & 12 & \\
HI $(\mathrm{N}=16)$ & 6 & 0 & \\
$\mathrm{I}(\mathrm{N}=24)$ & 20 & 4 & \\
\hline \multicolumn{4}{r}{ Table 2. Association of MP3 Stages with Menarche } \\
\hline
\end{tabular}

\begin{tabular}{|cccc|}
\hline Menarche & Yes & No & $\times 2$-value \\
$\mathrm{E}(\mathrm{n}=15)$ & 0 & 15 & \\
$\mathrm{~F}(\mathrm{n}=35)$ & 1 & 34 & 45.68 \\
$\mathrm{G}(\mathrm{n}=21)$ & 7 & 14 & $\mathrm{p}=0.0001, \mathrm{~S}$ \\
$\mathrm{H}(\mathrm{n}=69)$ & 43 & 26 & \\
\hline \multicolumn{4}{|r|}{ Table 3. Association of Canine Stages with Menarche } \\
\hline
\end{tabular}

\begin{tabular}{|c|c|c|c|c|c|c|c|}
\hline$\stackrel{m}{\Sigma}$ & 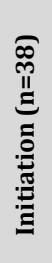 & 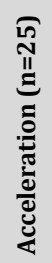 & 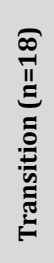 & 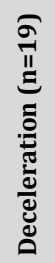 & 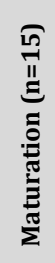 & 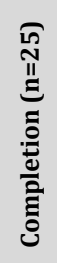 & 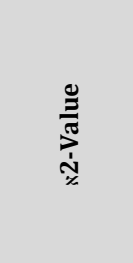 \\
\hline F & 38 & 2 & 0 & 0 & 0 & 0 & \\
\hline $\mathrm{FG}$ & 0 & 22 & 1 & 0 & 0 & 0 & \\
\hline G & 0 & 0 & 17 & 0 & 0 & 0 & 643.24 \\
\hline $\mathrm{H}$ & 0 & 1 & 0 & 19 & 0 & 0 & $P=0.0001, S$ \\
\hline $\mathrm{HI}$ & 0 & 0 & 0 & 0 & 15 & 1 & \\
\hline I & 0 & 0 & 0 & 0 & 0 & 24 & \\
\hline \multicolumn{8}{|c|}{ Table 4. Association of CVM Status with MP3 Stages } \\
\hline
\end{tabular}

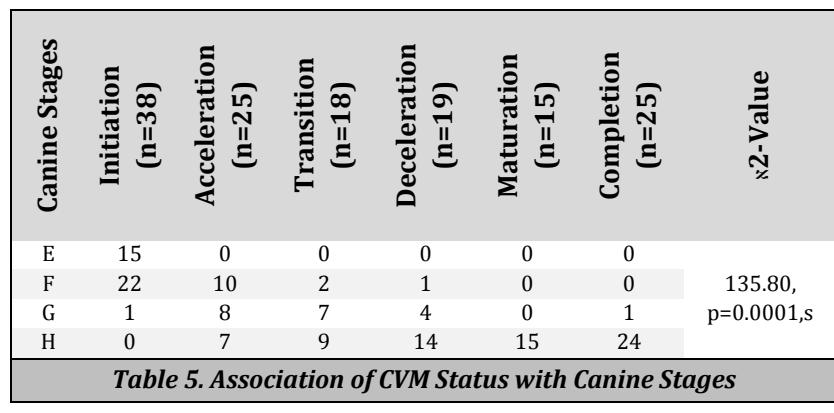

\begin{tabular}{|cccccc|}
\hline MP3 & E (n=15) & F (n=35) & G (n=21) & H (n=69) & *2-value \\
F & 15 & 23 & 2 & 0 & \\
FG & 0 & 8 & 8 & 7 & \\
G & 0 & 2 & 6 & 9 & 86.97 \\
H & 0 & 2 & 4 & 14 & $\mathrm{P}=0.0001, \mathrm{~s}$ \\
HI & 0 & 0 & 1 & 15 & \\
I & 0 & 0 & 0 & 24 & \\
\hline \multicolumn{5}{r}{ Table 6. Association of Canine Status with MP3 Stages } \\
\hline
\end{tabular}

\section{DISCUSSION}

The knowledge regarding growth and development is useful when patients are advised for orthopaedic or orthodontic treatment, as few phases of pubertal growth spurt may help the treatment of some types of malocclusion related with skeletal disorders. ${ }^{27}$ When growth prediction is done before an orthodontic treatment it will directly help in orthodontic diagnosis, planning and prognosis; 28,29 predominantly as growth increments are exploited during this phase. ${ }^{29}$

Females typically mature early, with a mean difference of two years for the onset of pubertal growth spurt.29,30 Boys not only reach maturation late, but the scale of their growth velocity peak is also noticeably superior than that of females. Hagg and Taranger (1982) proposed that if menarche has happened, peak height velocity has been achieved and growth rate is decelerating. This propose that all the treatment which are associated to growth, chiefly the modification of skeletal discrepancy need to be commenced before menarche to attain most serious time for the orthopaedic correction of jaw. Clinically, these alterations recommend the need to start orthodontic treatment earlier in females than in boys. ${ }^{29}$

Skeletal maturation, the utmost common index in clinical routine, is thought to be related with sexual and physical maturation. ${ }^{23}$ Profound hormonal changes are perceived during puberty. ${ }^{29}$ These hormones have a metabolic effect, which encourages bone growth and rules the development of the primary and secondary sexual traits. ${ }^{31}$ There is a persistent association between menarche (first menstruation) and the time at which pubertal growth peak happens. Height velocity peak is stretched about one year before menarche, and is an exceptional guideline to measure the sexual maturation of females. $3,23,29$

On correlation of CVM status with menarche, it was found that there was no onset of menstruation in initiation stage. An acceleration stage signified the onset of menstruation in $8 \%$ of females. This percentage was found to be increasing as CVM stage progressed further. All the females attending maturation stage had menarche. However, in the completion stage of CVMI, 84\% showed of onset of menstruation. Mehrnaz Moradinejad (2016) ${ }^{32}$ stated that highest percentage of the incidence of menarche was related to the 
fourth stage of CVM (37.5\%), with the minimum in the first stage $(0 \%)$. The highest incidence rates of menarche were noted in the fourth and fifth stages of the development of cervical vertebrae. Eddie Hsiang-Hua Lai (2008) ${ }^{33}$ calculated relationship between age at menarche and skeletal maturation stages in Taiwanese female Orthodontic patients. They determined that the majority of female orthodontic patients have attained the pubertal growth spurt when they experienced their menarche all the findings suggest that menarche typically trails the pubertal growth spurt by about 1 year and ensues after CVMS III. This suggests that the majority of female orthodontic patients would experience menarche by the time adolescent growth decelerates intensely and before the growth spurt is accomplished. It is thereby recommended that the orthopaedic treatment for females should be started at the acceleration stage of CVMI.

When MP3 was correlated with menarche it was observed that no females in MP3 F stage had achieved menarche while maximum number of females in $\mathrm{HI}$ and I stage reached menarche. Minimum number of females have onset of menstruation in FG and G stage. With the onset of menstruation increasing progressively with the different stage of MP3. These differences were statistically significant $(\mathrm{p}<0.05)$. There are very few studies quoting the correlation between MP3 and menarche. Those results can be considered as newer information for the growth-related studies to evaluate the pubertal status.

When developmental stage of canine was correlated with menarche, it was found that $100 \%$ of the patients with canine stage $\mathrm{E}$ did not have onset of menstruation while maximum number of patients with canine stage $\mathrm{H}$ had menarche followed by $G$ and $F$ stage respectively. This suggested that orthopaedic treatment should be started before stage $\mathrm{H}$. Similar result was suggested by Alizé Lacoste Jeanson et al (2013). ${ }^{34}$ Bayesian interpretation supports the results formerly obtained by giving probability of attribution for each mineralization sequence in ante, peri and postmenarche groups. It seemed that the development of dental mineralization is interrelated to menarche.

When the results were correlated it was seen that initiation stage of CVM correlated with the MP3F, acceleration stage of CVM with MP3FG, Transition stage of CVM with MP3G, deceleration stage of CVM with MP3H, maturation stage of CVM with MP3HI and completion stage of CVM with MP3I. There remained a high statistically significant variance amongst various stages $(\mathrm{p}<0.05)$ suggesting a directly proportional relation of MP3 \& CVM stages with the advancing chronological age.

Mandava Prasad et al (2013) ${ }^{35}$ also stated a decent concordance among six stages of CVM (Hassel and Farman ${ }^{18}$ ) and the six stages of MP3 (Rajagopal and Kansal ${ }^{16}$ ). Santiago et al $(2012)^{36}$ reviewed the literature regarding the reliability of CVM stages to predict the pubertal spurt and found a moderate to high statistically significant connection among CVM and hand-wrist maturation methods. There was a moderate to high reproducibility of the CVM method, and only one precise study explored the exactness of the CVM index in distinguishing peak pubertal growth.

When CVM was correlated to canine development, initiation and acceleration stage of CVM correlates with $\mathrm{F}$ stage of canine development, transition and deceleration stage of CVM correlates with the $\mathrm{H}$ stage of canine development, whereas, maturation and completion stage of CVM development correlates with $\mathrm{H}$ stage. There was a statistically significant difference $(\mathrm{p}<0.05)$. Ingrid RóżyłoKalinowska $(2016)^{37}$ also specified the utility of dental calcification stages as a modest first-level diagnostic test to regulate the skeletal maturity status. MSV Kishore (2016) ${ }^{38}$ also established that both dental and skeletal maturity should be measured if the maturity stage of a growing child is to be pertinent to clinical practice.

When E stage of canine development was correlated with stage of MP3 development, it coincided 100\% with F stage of MP3 development. The F stage of canine development coincided $65.71 \%$ with F stage of MP3 development followed by FG stage of MP3 development. The G stage of canine development correlate $38.10 \%$ with FG stage of MP3 development followed by $\mathrm{G}$ and $\mathrm{H}$ stage $28.57 \%, 19.05 \%$ respectively. The $\mathrm{H}$ stage of canine development coincides maximally (34.78\%) with I stage of MP3 development stage followed by $\mathrm{HI}$ and $\mathrm{H}$ stage of MP3 development. These differences were statistically significant $(p<0.05)$. Reshma Nayak et al $(2010)^{39}$ in their study revealed that the maturation of the middle phalanx of third finger and the mandibular canine progress with advancing age. There are progressively cumulative stages of MP3 stages and canine development stages with age.

Surendran and Thomas et al (2014) ${ }^{40}$ stated that Dental maturation assessment is in an important tool to analyse prepubertal and post pubertal growth phases. The result of correlation between canine calcification stages and MP3 stages were highly statistically significant.

The developmental stages of the mandibular canine can be used as an indicator in assessing the skeletal maturity. The relation between mandibular canine development and MP3 stages also permits the clinician to recognize the early stages of the pubertal growth spurt. According to United States reference data the initiation of the spurt is showed by canine stage F. Stage G, overlaps with the eruption of the canine into the oral cavity, which occurs roughly one year before the peak height velocity in boys, but 5 months before in females. This may imitate hormonal changes which accompany puberty.

\section{CONCLUSIONS}

CVM stages shows that skeletal maturation is completed by the age of 14 years in females which coincides with peak in growth at around 10 - 11 years of age, it suggests that growth modification procedure for maxillary arch must be carried out 1 to 2 years before peak growth in females takes place. There is a strong correlation between CVMI and MP3, so one of the two methods can be used for skeletal age assessment. Mandibular canine can be used to evaluate skeletal age and can be used as dependable indicator for assessment of pubertal growth status of an individual. Skeletal maturity indicators such as CVM status and MP3 we studied was found to be ahead of chronological age There is strong correlation between all the parameters such as biological age, skeletal age, and dental age and all these are ahead of chronological age especially dental age. That is, age is not the parameter to decide interception using growth modulation. 
There are strong correlations between all the parameters studied which signifies that assessment of skeletal maturation with any of the above methods studied, gives exact growth maturation age of the patient. There is significant early onset in the age of menarche and skeletal age, which implies that there is a need to start the orthopaedic treatment related to growth modification at an early age in females. There is a paradigm shift in the change in the timing of growth tenants which has been pretimed in males and females. Early onset of menstruation suggests that females tend to mature and complete their skeletal growth earlier than males, therefore, growth modification procedure should be initiated early in females as the early menarche can be correlated to the secular trends. The identification of growth should be initiated at an earlier age in females than that described in literature.

From above study, it is suggested that with consideration to the orthopedic changes, two stage therapy and growth modification for females, due to early maturation, should be initiated at early stage.

\section{REFERENCES}

[1] Proffit WR, Fields HW, Sarver DM. Contemporary orthodontics. $4^{\text {th }}$ edn. St. Louis, Mo: Mosby Elsevier 2007.

[2] Srinivasan B, Premkumar S. Assessment of serum dehydroepiandrosteronesulphate in subjects during the pre-pubertal, pubertal and adult stages of skeletal maturation. Eur J Orthod 2012;34(4):447-51.

[3] Nanda RS. The rates of growth of several facial components measured from serial cephalometric roentgenograms. Am J Orthod Dentofacial Orthop 1955;41(9):658-73.

[4] Björk A. Variations in the growth pattern of the human mandible: longitudinal radiographic study by the implant method. J Dent Res 1963;42(1 Pt 2):400-11.

[5] Hunter CJ. The correlation of facial growth with body height and skeletal maturation at adolescence. The Angle Orthodontist 1966;36(1):44-54.

[6] Greulich WW, Pyle SI. Radiographic atlas of skeletal development of the hand and wrist. $2^{\text {nd }}$ edn. Stanford University Press 1959.

[7] Björk A, Helm S. Prediction of the age of maximum puberal growth in body height. Angle Orthod 1967;37(2):134-43.

[8] Tofani MI. Mandibular growth at puberty. Am J Orthod Dentofacial Orthop 1972;62(2):176-95.

[9] Hägg U, Taranger J. Menarche and voice changes as indicators of the pubertal growth spurt. Acta Odontol Scand 1980;38(3):179-86.

[10] Tanner JM. Growth at adolescence. $2^{\text {nd }}$ edn. Oxford: Blackwell Science 1962.

[11] Hägg U, Taranger J. Skeletal stages of the hand and wrist as indicators of the pubertal growth spurt. Acta Odontol Scand 1980;38(3):187-200.

[12] Baccetti T, Franchi L, McNamara JA. The Cervical Vertebral Maturation (CVM) method for the assessment of optimal treatment timing in dentofacial orthopedics. Seminars in Orthodontics 2005;11(3):119-29.
[13] Mitchell L. An introduction to orthodontics. $3^{\text {rd }}$ edn. UK: Oxford University Press 2007.

[14] Flores-Mir C, Nebbe B, Major PW. Use of skeletal maturation based on hand wrist radiographanalysis as predictor of facial growth: a systematic review. Angle Orthod 2004;74(1):118-24.

[15] Hagg U, Taranger J. Maturation indicators and pubertal growth spurt. Am J Orthod 1982;82(4):299-309.

[16] Rajagopal R, Kansal S. A comparison of modified Mp3 stages and cervical vertebrae as growth indicators. J Clin Orthod 2002;36(7):398-406.

[17] Lamparski DG. Skeletal age assessment utilizing cervical vertebrae. Master of Science Thesis, University of Pittsburg, Pittsburg. 1972.

[18] Hassel B, Farman AG. Skeletal maturation evaluation using cervical vertebrae. Am J Orthod Dentofacial Orthop 1995;107(1):58-66.

[19] Moorrees CF, Fanning EA, Hunt EE Jr. Age variation of formation stages for ten permanent teeth. J Dent Res 1963;42:1490-502.

[20] Nolla CM. The Development of permanent teeth. J Dent Child 1960.

[21] Anderson DL, Thompson GW, Popovich F. Interrelationships of dental maturity, skeletal maturity, height and weight from age 4 To 14 years. Growth 1975;39(4):453-62.

[22] Liliequist B, Lundberg M. Skeletal and tooth development. A methodologic investigation. Acta Radiol Diagn (Stockh) 1971;11(2):97-112.

[23] Demirjian A, Buschang PH, Tanguay $\mathrm{R}$, et al. Interrelationships among measures of somatic, skeletal, dental and sexual maturity. Am J Orthod 1985;88(5):433-8.

[24] Chertkow S, Fatti P. The relationship between tooth mineralization and early radiographic evidence of the ulnar sesamoid. Angle Orthodont 1979;49(4):282-8.

[25] Chertkow S. Tooth mineralization as an indicator of the pubertal growth spurt. Am J Orthod 1980;77(1):79-91.

[26] Sierra AM. Assessment of dental and skeletal maturity - a new approach. Angle Orthodontist 1987;57(3):194-208.

[27] Houston WJ, Miller JC, Tanner JM. Prediction of the timing of the adolescent growth spurt from ossification events in hand-wrist films. Br J Orthod 1979;6(3):14552.

[28] Ruf S, Pancherz H. Development of the frontal sinus in relation to somatic and skeletal maturity. A cephalometric roentgenographic study at puberty. Eur J Orthod 1996;18(5):491-7.

[29] Burtone CJ. Process of maturation and growth prediction. Am J Orthod 1963;49(12):907-19.

[30] Tibério S, Vigorito JW. 0 estudo da maturação esquelética de crianças brasileiras de 08 a 15 anos, em referência à ossificação dos ossos psiforme, ganchoso, falanges média e proximal dos dedos 2 e 3. Ortodontia 1989;22(2):4-19.

[31] Coutinho S, Buschang PH, Miranda F. Relationships between mandibular canine calcification stages and skeletal maturity. Am J Orthod Dentofacial Orthop 1993;104(3):262-8.

[32] Moradinejad M, Berahman N, Sadeghi E, et al. Determination of developmental stage of cervical vertebrae at menarche in female orthodontic patients in 
Ahwaz. Int J Med Res \& Health Sci 2016;5(8):206-10.

[33] Eddie HHL, Jenny ZCC, Yao CCJ, et al. Relationship between age at menarche and skeletal maturation stages in Taiwanese female orthodontic patients. J Formos Med Assoc 2008;107(7):527-32.

[34] Jeanson AL, Urzel V, Bruzek J, et al. Dental mineralization as an indicator of sexual maturity in girls. J Forensic Odonto Stomatology 2013;31(Suppl 1):57-8.

[35] Prasad M, Ganji VSK, George SA, et al. A comparison between cervical vertebrae and modified MP3 stages for the assessment of skeletal maturity. Journal of Natural Science, Biology and Medicine 2013;4(1):74-80.

[36] Santiago RC, de Miranda CLF, Vitral RWF, et al. Cervical vertebral maturation as a biologic indicator of skeletal maturity. Angle Orthod 2012;82(6):1123-31.
[37] Ingrid RK, Anna KR, Pawel K. Relationship between dental age according to Demirjian and cervical vertebrae maturity in polish children. European Journal of Orthodontics 2011;33(1):75-83.

[38] Kishore MSV, Sravya RL, Dasari AK, et al. Diagnostic potential of mandibular second premolar and its relation to physiological and skeletal maturity in girls. Int J Dentistry Oral Sci 2016;3(7):291-5.

[39] Nayak R, Nayak USK, Hegde G. Assessment of growth using mandibular canine calcification stages and its correlation with modified MP3 stages. International Journal of Clinical Pediatric Dentistry 2010;3(1):27-33.

[40] Surendran S, Thomas E. Tooth mineralization stages as a diagnostic tool for assessment of skeletal maturity. Am J Orthod Dentofacial Orthop 2014;145(1):7-14. 